\title{
Temperature Dependence of Upper Critical Field as an Indicator of boson Effects in Superconductivity in $\mathrm{Nd}_{2-x} \mathrm{Ce}_{x} \mathrm{CuO}_{4-y}$
}

\author{
V. F. Gantmakher *, G. A. Emel'chenko, I. G. Naumenko, and G. E. Tsydynzhapov \\ Institute of Solid State Physics RAS, 142432 Chernogolovka, Russia
}

The temperature dependence of upper critical field $B_{c 2}$ was determined from the shift of resistive transition $\Delta T(B)$ in nearly optimally doped $\mathrm{Nd}_{2-x} \mathrm{Ce}_{x} \mathrm{CuO}_{4-y}$ single crystals. Within the experimental accuracy, the weak-field data are described by power function $B_{c 2} \propto(\Delta T)^{3 / 2}$. This result is compared with the data on heat capacity and analyzed in the context of possible manifestations of boson effects in superconductivity. The $T$ dependence of $B_{c 2}$ persists down to the lowest temperatures, but the numerical values of $B_{c 2}$ below 1 $\mathrm{K}$ are different for different samples.

PACS numbers: 74.20.Mn, 74.25.Dw, 74.72.-h.22

There are grounds to believe that high-temperature superconductivity (HTSC) is not described by the BCS theory. One of them consists in the relationship between the density $n$ of Cooper pairs and the coherence length $\xi$ (the pair size). In HTSC cuprates, superconductivity is due to the carriers in the $\mathrm{CuO}_{2}$ plane. Like in all 2D systems, the density of states $g_{F}$ at the Fermi level in the $\mathrm{CuO}_{2}$ plane does not depend on the carrier concentration in the normal state and, according to measurements, is equal to $g_{F}=2.5 \cdot 10^{-4} \mathrm{~K}^{-1}$ per one structural unit of $\mathrm{CuO}_{2}$ (this value is nearly the same for all cuprate families, see, e.g., [1], Ch. 13). Assuming that the superconducting gap $\Delta$ is of the order of transition temperature $T_{c}$, one estimates the mean distance $r=n^{-1 / 2} \approx\left(g_{F} \Delta\right)^{-1 / 2}$ between the pairs in the $\mathrm{CuO}_{2}$ plane at $25 \AA$ for $T_{c} \approx 100 \mathrm{~K}$ and $75 \AA$ for $T_{c} \approx 10 \mathrm{~K}$. These $r$ values should be compared with the typical coherence length $\xi \approx 20 \AA$ in the $a b$ plane [1], so that $r \gtrsim \xi$ in the HTSC materials. Inasmuch as the BCS theory introduces Cooper pairs to describe the Fermi-liquid ground state as a whole, its validity for the description of HTSC is not obvious. This causes interest in the models of Bose superconductivity for which $r \gg \xi$ and which are based on Bose-Einstein condensation (BEC) in a system of charged bosons [2-4]. The experimental evidences for the boson effects in HTSC are presently intensively accumulated.

One such evidence can be expected to obtain from the measurements of the temperature dependence of magnetic field $B_{c 2}$ destroying superconductivity. In the BCS theory, the $B_{c 2}\left(T / T_{c}\right) / B_{c 2}(0)$ function is linear in the vicinity of $T / T_{c}=1$; it monotonically increases to saturation near the zero temperature and almost coincides with the limiting value even at $T / T_{c}=0.2[5]$. However, in most cases, the HTSC materials behave in a different manner and demonstrate positive second derivative $\partial^{2} B_{c 2} / \partial T^{2}$ over the entire temperature range.

The $B_{c 2}(T)$ measurements are mainly based on an analysis of the resistive transition. Two types of behavior are known for the resistive transition of HTSC materials in a magnetic field. For one of them, the transition is sizably broadened in a magnetic field, so that it is hard and even practically impossible to gain from it any information about the $B_{c 2}(T)$ dependence. The other transition is shifted in a magnetic field to lower temperatures and either remains undistorted, as in usual superconductors, or undergoes an insignificant distortion. This usually occurs for those members of HTSC families in which $T_{c} \leq 20 \mathrm{~K}$. The transition shift in these materials is naturally explained by the field-induced destruction of superconductivity. Irrespective of the mechanism of dissipative processes in the superconducting state, the spectrum rearrangement and the appearance of superconducting pairing should necessarily affect the $R(T)$ resistance. With this preposition, one can readily construct the $B_{c 2}(T)$ function.

Almost in all HTSC cuprates such as the Tl-based [6] and Bi- based [7] families and the LaSrCuO [8] and $\mathrm{Nd}(\mathrm{Sm}) \mathrm{CeCuO}[9-11]$ families, as well as in the Zn-doped [12] or oxygen-deficient [13] $\mathrm{YBaCuO}$, the $B_{c 2}(T)$ function derived from the shift of resistive transition has the positive second derivative over the whole temperature range $0<T / T_{c}<1$ and shows a tendency to diverge at small $T / T_{c}$ values. Most discussion over the $B_{c 2}(T)$ curves concentrated precisely on this divergence and considered it as the most dramatic departure from the BCS theory. At the same time, the behavior of the $B_{c 2}(T)$ function near $T_{c}$ is also quite informative. Contrary to expectations, almost in all cases where the field-induced resistive-transition shift in HTSC cuprates pro-ceeds in a parallel manner, the experimental data indicate that the $\partial B_{c 2} / \partial T$ derivative is zero at the $T_{c}$ point [6-13].

The $\partial B_{c} / \partial T$ derivative of critical field in the $T_{c}$ point is related to the free energy $F$ and heat capacity $C$ in this point by the well-known Rutgers formula:

$$
\frac{1}{4 \pi}\left(\frac{\partial B_{c}}{\partial T}\right)_{T_{c}}^{2}=\frac{\partial^{2}}{\partial T^{2}}\left(F_{s}-F_{n}\right)=\frac{C_{s}-C_{n}}{T_{c}} .
$$

Inasmuch as the thermodynamic critical field $B_{c}$ is different from the upper critical field $B_{c 2}$, Eq. (1) can be used only for qualitative estimates. However, being based on thermodynamics, this equation is very useful.

In usual superconductors, $F_{s}-F_{n} \propto\left(T_{c}-T\right)^{2}$, so that the heat capacity undergoes a jump and $B_{c}$ is linear in $\left(T_{c}-T\right)$. In the BEC case, $F_{s}-F_{n} \propto\left(T_{c}-T\right)^{3}$, so that the heat capacity is a continuous function in the 
transition point [14]. It then immediately follows that $\partial B_{c} / \partial T=0$ and

$$
B_{c} \propto\left(T_{c}-T\right)^{3 / 2} .
$$

Of course, one can hardly imagine that the Fermi gas suddenly and completely transforms into a Bose gas at low temperatures. It was assumed in [4] that bosons appear in small pockets of the $\mathbf{k}$-space near the Fermi level. In the isotropic model, one can only speak about pairing of sufficiently energetic fermions, as in the BCS theory. This kind of model has been proposed in [15]. Nevertheless, Eq. (2) deserves a serious experimental verification.

Such was the motivation of our work consisting in the measurement and analysis of the field-induced shift of resistive transition in $\mathrm{Nd}_{2-x} \mathrm{Ce}_{x} \mathrm{CuO}_{4-y}$ single crystals. We will discuss separately the behavior of the $B_{c 2}$ field in the vicinity of $T_{c}$ and at low temperatures.

Experiment. $(\mathrm{NdCe})_{2} \mathrm{CuO}_{4}$ single crystals were grown from a mixture of components taken in the molar ratio $\mathrm{Nd}_{2} \mathrm{O}_{3}: \mathrm{CeO}_{2}: \mathrm{CuO}=1: 0.05: 11$ in a crucible made from yttrium-stabilized zirconium dioxide. The use of a modified growth regime markedly reduced the time of interaction between the melt and the crucible at high temperature. Owing to the accelerated-decelerated crucible rotation, the melt was intensively stirred so that the homogenization time for the molten solution did not exceed $1 \mathrm{~h}$ at a temperature near $1150{ }^{\circ} \mathrm{C}$. The growth was carried out for several hours upon slow cooling ( 6 $\mathrm{K} / \mathrm{h}$ ) under the conditions of morphologically stable crystallization front $(d T / d x \geq 10 \mathrm{~K} / \mathrm{cm})$, after which the crucible was decanted and cooled at a rate of 30-50 $\mathrm{K} / \mathrm{h}$ to ambient temperature. The crystals were shaped like platelets of thickness $20-40 \mathrm{~mm}$. Their composition $\mathrm{Nd}_{1.82} \mathrm{Ce}_{0.18} \mathrm{CuO}_{x}$ was determined by local X-ray spectroscopic analysis. The analysis revealed $\mathrm{Zn}$ traces in the crystals at a level of $0.1 \mathrm{wt} \%$. Initially the crystals did not show superconducting transition above $4.2 \mathrm{~K}$. The superconducting transition at $T_{c} \approx 20 \mathrm{~K}$ appeared after 15 -h annealing at $900{ }^{\circ} \mathrm{C}$ in an argon atmosphere.

Measurements were made for two plates approximately $1 \times 2 \mathrm{~mm}$ in size. The silver paste contacts were fused in the air at a temperature of $350{ }^{\circ} \mathrm{C}$. Four contacts in sample 1 were arranged $0.5 \mathrm{~mm}$ apart in a row on one side of the plate. The potential contacts in sample 2 were placed on the opposite side of the plate beneath the current contacts, allowing the measuring current to be directed both along and transversely the ab plane. This did not affect the results. The resistance was measured by the standard method using a lock-in nanovoltmeter at a frequency of $13 \mathrm{~Hz}$. The measuring current was small enough for the linear regime and the absence of overheating to be provided down to the lowest temperatures. The magnetic field was directed along the normal to the plate ( $c$ axis). Measurements were performed over the temper-

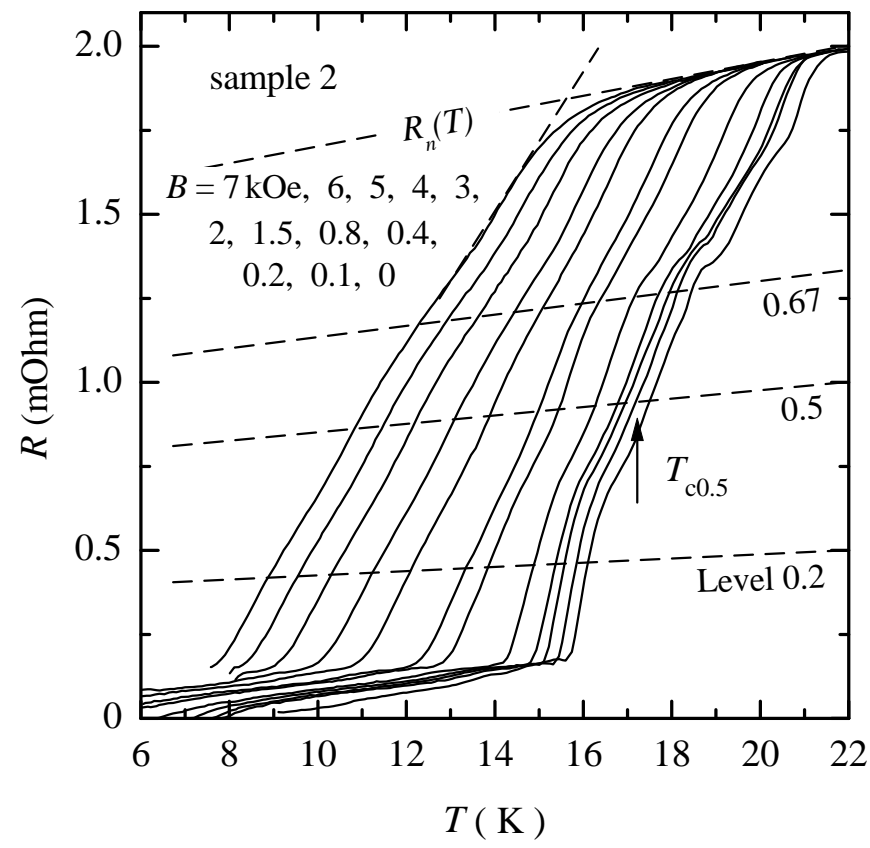

FIG. 1. The $R(T)$ curves for sample 2 in magnetic fields (from right to left) from 0 to $7 \mathrm{kOe}$. The dashed lines are the straight line $R_{n}(T)$ and the straight lines at the levels of 0.67 , 0.5 , and 0.2 of $R_{n}(T)$. The method of determining the onset of transition is demonstrated and the $T_{c i}$ fields from which the shifts were measured are shown

ature range from $25 \mathrm{~K}$ to $25 \mathrm{mK}$. T The onset of zero-field superconducting transition in both samples occurred at about $20.5 \mathrm{~K}$.

The measurements gave identical results for both samples. Figure 1 demonstrates a series of low-field $R(T)$ curves for sample 2. At high temperatures, all curves show the same asymptotic behavior $R_{n}(T)$ above the transition, and one can assume that the $R_{n}$ function does not depend on $B$ at $T>10-12 \mathrm{~K}$. The zero-field transition shows a certain structure, which, however, is smoothed out even at 100-200 Oe. The field effect mainly amounts to shifting the transition to lower temperatures. The degree in which this shift is parallel can be checked by comparing the shift of the onset of transition with the shifts of the $R(T)$ function at different levels: $0.2 R_{n}$, $0.5 R_{n}$, and $0.67 R_{n}$ (see curves in Fig. 1). For the parallel shift, all constructions in Fig. 1 should give the same function $B_{c 2}(\Delta T)$, where $\Delta T=T_{c i}-T$ and $T_{c i}$ is the temperature corresponding to the same level on the initial curve $R(T, B=0)$. The log-log plots of the shifts are shown by different symbols in Fig. 2a for all four levels. The systematic deviations of the symbols from the

\footnotetext{
${ }^{1}$ The low-temperature measurements in strong magnetic fields were carried out at the NHMFL (Tallahassee, Fla., USA).
} 

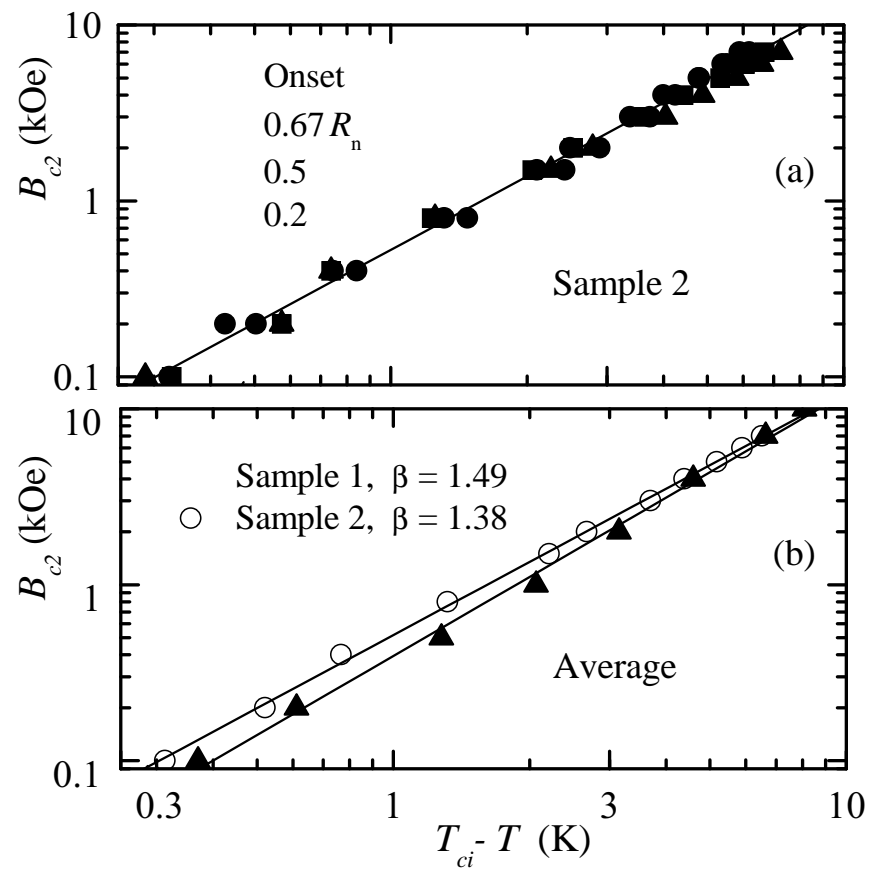

FIG. 2. (a) Plots of the field vs. shift at different levels in this field; (b) the same for the averaged shifts for two samples.

straight line

$$
B_{c 2}=(\Delta T)^{\beta},
$$

constructed by averaging the results for all points are small for each of the symbols. This implies that the distortions of the transition shape are small as compared to its shift. The scatter of points in low fields is mainly caused by the fine structure of the $R(T, B=0)$ curve, which serves as a reference in the determination of the shift $\Delta T$.

The coefficient $\beta$ was determined from the slope of the straight line passing through the averaged $\Delta T$ shifts (Fig. $2 \mathrm{~b}$ ). The curve processing for sample 2 (Fig. 1) yields $\beta \approx 1.4$, and the processing of analogous curves for sample 1 yields $\beta \approx 1.5$.

The resistances for both crystals decreased in a relatively narrow temperature range not to zero; one can see in Fig. 1 that, starting at the level of $\sim 0.1$, a slanting tail appears. The same tail for sample 1 starts at a higher level of $\sim 0.2$. In this work, we will analyze only the upper portion of the transition, assuming that the electron spectrum is rearranged into the form characteristic of the superconducting state precisely in this region.

Figure 3 shows the $R(B)$ functions for very low temperatures $T / T_{c 0}<0.05$. In this region, the normal resistance depends, though weakly, on a magnetic field, while the onset of transition is clearly defined and its shift is easily detected even upon changing temperature below $T / T_{c 0}=0.005$. When considering the $B_{c 2}(T)$ functions in this region (see inset in Fig. 3), two fact are noteworthy. First, $B_{c 2}$ does not show tendency to diverge near

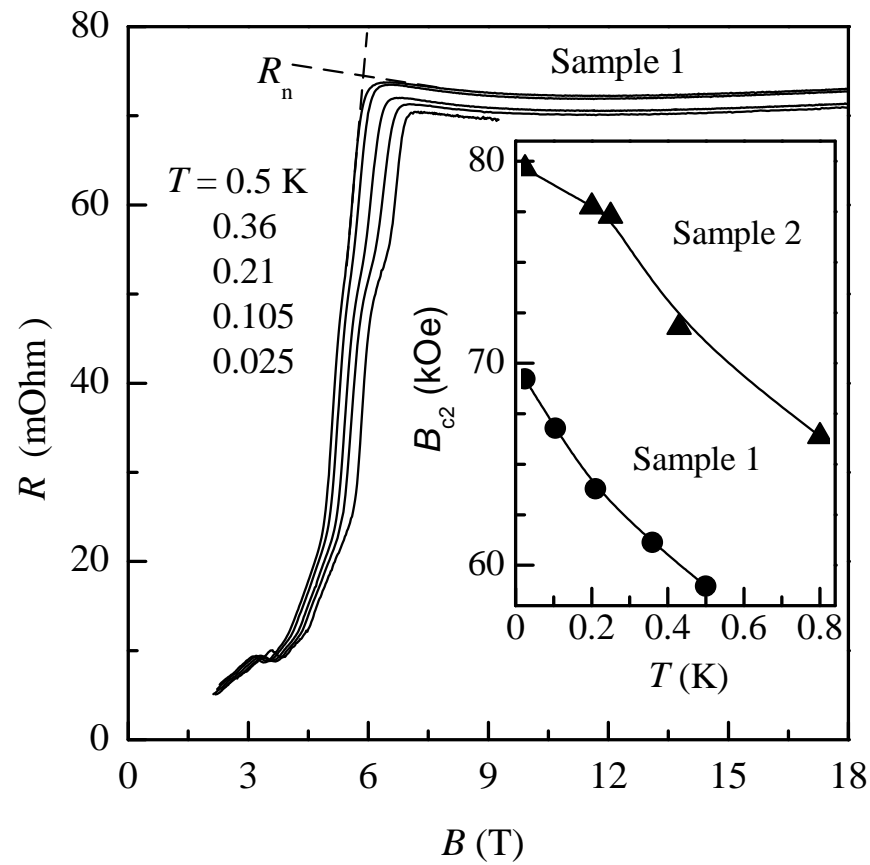

FIG. 3. The $R(B)$ curves for sample 1 at temperatures (from left to right) from $0.5 \mathrm{~K}$ to $25 \mathrm{mK}$. Inset: the field of the onset of transition at low temperatures for both samples.

zero temperature; although the derivative of $B_{c 2}(T)$ is large below $0.5 \mathrm{~K}$, the function is linear within the experimental accuracy and extrapolated to a finite value $B_{c 2}(0)$ (similar result was obtained previously for thallium crystals [6]). Second, the critical fields at low temperature are equal to 69 and $80 \mathrm{kOe}$ for samples 1 and 2 , respectively, i.e., differ by more than $10 \%$, inspite of the fact that the crystals were from the same batch and their $T_{c}$ values coincide.

The graph of $B_{c 2}(T)$ over the entire temperature range is shown in the inset in Fig. 4; as in other HTSC cuprates, the second derivative $\partial^{2} B_{c 2} / \partial T^{2} \geq 0$ for all temperatures (cf., e.g., [6, 7]).

Discussion. It follows from the preceding section that our data for the vicinity of $T_{c}$ are consistent, within the experimental accuracy, with Eq. (2). It would have been instructive to compare these data with the data on heat capacity, but, unfortunately, in the works where the heat capacity of $\mathrm{Nd}_{2-x} \mathrm{Ce}_{x} \mathrm{CuO}_{4-y}$ was measured [16] the contribution of critical fluctuations near $T_{c}$ was not determined. Nevertheless, it is known that the measurements of heat capacity of the HTSC materials show strong dissimilarity from usual superconductors [17] but do not allow the discrimination between the BCS and BEC models. These problems can be illustrated by comparing the results of measurements of the resistance and heat capacity of the thallium high- $T_{c}$ superconductor. No explicit jump in heat capacity is observed for this compound even at zero field, although the contribution from the critical fluctuations is undoubtedly present in the tempera- 


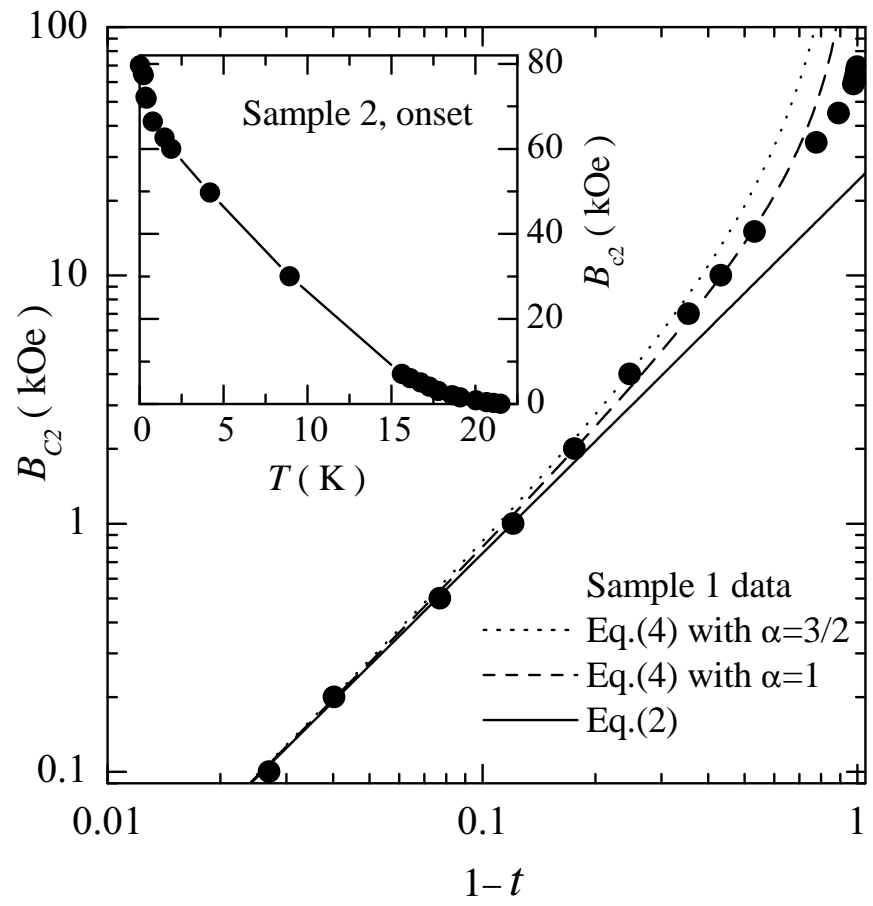

FIG. 4. Comparison of the experimental $B_{c 2}$ values for sample 1 with Eqs. (2) and (4). Inset: the $B_{c 2}(T)$ function for sample 2 in the whole temperature region. The line is a guide to the eye.

ture range 16-10 $\mathrm{K}$ [18]; this contribution is reduced by approximately one-half in a field of $0.4 \mathrm{~T}$ and remains virtually in the same temperature range. At the same time, the resistive measurements by the same experimental group [6] suggest that a field of $0.4 \mathrm{~T}$ shifts the transition by $25 \%$ from 16 to $12 \mathrm{~K}$.

In connection with this contradiction, an interesting remark was made in [19], where the numerical calculations were carried out for the heat capacity of an ideal charged Bose gas in a weak magnetic field. It is well-known that the BEC does not occur in the ideal charged Bose gas in a uniform magnetic field [20] because the density of states diverges at the lower Landau level of the spectra of charged bosons. This implies that the transition occurs only at an isolated point in the $(T, B)$ plane. The magnetic field in this plane is scaled by the comparison of the cyclotron energy $\hbar e B / m c$ with $T_{c}$. Substituting the free electron charge and mass for $e$ and $m$, respectively, one arrives at the value of $8 \mathrm{~T}$ for the characteristic field at $T_{c}=16 \mathrm{~K}$. On this scale, the above-mentioned field of $0.4 \mathrm{~T}$ is as small as 0.05 . As long as the field is low, the phase trajectory again passes through the vicinity of the transition point in the $(T, B)$ plane upon changing $T$, but, as the field increases, the "impact parameter" increases, while the contribution of critical fluctuations decreases. However, the temperature interval corresponding to the small impact parameters does not change. In the case that the transition is the BEC in a weakly non- ideal charged Bose gas, this contribution is hidden from view at the lower temperature where the transition occurs in magnetic field. Then, strange as it may seem, the resistive measurements provide the more reliable information on the transition position than the heat capacity measurements do.

According to the results obtained for the immediate vicinity of $T_{c}$, the behavior of the $B_{c 2}(T)$ function should be compared with the predictions of the superconductivity models in a nonideal Bose gas. Due to the boson scattering by impurities or to the boson-boson interaction, the critical field in a weakly nonideal Bose gas behaves as [21]

$$
B_{c 2} \propto t^{-\alpha}\left(1-t^{3 / 2}\right)^{3 / 2}, \quad t=T / T_{c},
$$

where, depending on the particular model, the exponent $\alpha$ is equal to 1 or $3 / 2[21,22]$. At $t \rightarrow 1$, function (4) takes the asymptotic form (2). It is seen in Fig. 4 that the experimental points deviate in the proper direction from the asymptote and, on the whole, correspond well to Eq. (4). A more detailed comparison is hardly pertinent, as long as the theories [21, 22] do not allow for the fieldinduced pair decay into fermions.

Conclusions. The field-induced distortion of the shape of resistive superconducting transition in the $\mathrm{Nd}_{2-x} \mathrm{Ce}_{x} \mathrm{CuO}_{4-y}$ single crystals is appreciably smaller than the transition shift. This allows the measurement of the $B_{c 2}(T)$ function. As zero-field $T_{c}$ is approached, the $B_{c 2}$ field behaves as a power function $B_{c 2} \propto(\Delta T)^{\beta}$ with $\beta \approx 1.5$ and, correspondingly, with a horizontal tangent $\partial B_{c 2} / \partial T=0$. This should imply the absence of jump in heat capacity at the zero-field phase transition. Such a behavior is precisely that which is expected for the heat capacity and critical field in the BEC of a charged Bose gas. For this reason, one of the possible conclusions that can be drawn from such a behavior of $B_{c 2}(T)$ near $T_{c}$ is that the description of superconductivity of the HTSC materials should involve the BEC elements, i.e., make allowance for the fact that fermions near the Fermi level tend to form bosons at temperatures above $T_{c}$. The $T$ dependence of $B_{c 2}$ persists down to the lowest temperatures, although, probably, the $B_{c 2}$ values in this region depend on lattice defects.

We are grateful to A.A. Abrikosov, L.P. Gor'kov, and V.P. Mineev for helpful discussions. The experiments at the NHMFL were performed in the framework of the program of cooperation between the NHMFL and scientists from the former USSR. This work was supported by the Russian Foundation for Basic Research (project no. 9902-16117), the Russian Foundation for Basic ResearchPICS (project no. 98-02-22037), the State Contract 107$2(00)-\mathrm{P}$, and the program "Statistical Physics" of the Ministry of Sciences of the Russian Federation. 
[1] J.R.Waldram, Superconductivity of Metals and Cuprates, Institute of Physics Publishing, 1996.

[2] V.Emery and S.Kivelson, Nature 374, 434 (1995).

[3] L.V.Ioffe and A.J.Millis, Phys. Rev. B 54, 3645 (1996).

[4] V.B.Geshkenbein, L.B.Ioffe, and A.I.Larkin, Phys. Rev. B 55, 3173 (1997).

[5] N.R.Werthamer, E.Helfand, and C.Hohenberg, Phys. Rev. 147, 295 (1966).

[6] A.P.Mackenzie, S.R.Julian, G.G.Lonzarich, et al., Phys. Rev. Lett 71, 1238 (1993); A.Carrington, A.P.Mackenzie, D.C.Sinclair, and J.P.Cooper, Phys. Rev. B 49, 13243 (1994).

[7] M.S.Osofsky, R.J.Soulen,Jr., S.A.Wolf, et al., Phys. Rev. Lett. 71, 2315 (1993); Journal of Supercond. 7, 279 (1994).

[8] M.Suzuki and M.Hikita, Jpn. Journal of Appl. Phys. 28, L1368 (1989).

[9] Y.Hidaka and M.Suzuki, Nature 338, 635 (1989); M.Suzuki and M.Hikita, Phys. Rev. B 41, 9566 (1990).

[10] Y.Dalichaouch, B.W.Lee, C.L.Seaman, et al., Phys. Rev. Lett. 64, 599 (1990).

[11] S.H.Han, C.C.Almasan, M.C.de Andrade, et al., Phys. Rev. B 46, 14290 (1992).

[12] D.J.C.Walker, O.Laborde, A.P.Mackenzie, et al., Phys. Rev. B 51, 9375 (1995).

[13] V. F. Gantmakher, G. E. Tsydynzhapov, L. P. Kozeeva, and A. N. Lavrov, Zh. Eksp. Teor. Fiz. 115, 268 (1999) [JETP 88, 148 (1999)].

[14] L. D. Landau and E. M. Lifshitz, Statistical Physics, (Nauka, Moscow, 1964; Pergamon, Oxford, 1980).

[15] J.Ranninger, J.M.Robin, and M.Eschrig, Phys. Rev. Lett. 74, 4027 (1995).

[16] E.Maiser, W.Mexner, R.Schäfer, et al., Phys. Rev. B 56, 12961 (1997).

[17] A.Junod, M.Roulin, B.Revaz, and A.Erb, Physica B 280, 214 (2000).

[18] A.Carrington, A.P.Mackenzie, and A.Tyler, Phys. Rev. B. 54, R3788 (1996).

[19] A.S.Alexandrov, W.H.Beere, V.V.Kabanov, and W.Y. Liang, Phys. Rev. Lett. 79, 1551 (1997).

[20] M.R.Shafroth, Phys.Rev. 100, 463 (1955).

[21] R.Micnas, J.Ranninger, and S.Robaszkievcz, Rev. Mod. Phys. 62, 113 (1990).

[22] A.S.Alexandrov, Phys. Rev. B 48, 10571 (1993). 